\title{
University of British Columbia
}

he Faculty of Forestry at the University of British Columbia (UBC) opened its newest professional degree program - the Master of International Forestry (MIF) - to applications on October 14, 2014. The MIF is designed for early/mid-career professionals (preferably $3+$ years of work experience) who wish to expedite their careers and advance to leadership positions. The program is targeted at individuals who want to engage productively-and to become leaders - in an international forestry context with international agencies and secretariats, finance institutions, transnational forest products enterprises, government ministries, consulting firms, environmental NGOs, and advocacy groups.

The MIF is a full-time, intensive 10month course-based master's degree that provides valuable skills relevant to a career in rural development, forest conservation planning, sustainable resource management, and conservation policy development, diplomacy and negotiation. Students in the MIF program will contend with the very immediate and complex interactions that occur between the political, economic, social and environmental spheres in a forestry context. Required courses include: international forest governance and policy; international forestry institutions, diplomacy and negotiations; natural resources economics; forest business enterprise; social, community and indigenous forestry; natural resources planning; and forests and society.

Students will be prepared for work in forestry in both developed and develop- ing countries, in natural and plantation forestry, and with subsistence and industrial forestry. In order to meet the rapidly changing needs of the international forestry job market, the MIF program will also provide training on those skills identified in over 200 forestry and environmental job postings. Immediately following the completion of course work in April 2016, students will undertake a short internship/directed study.

For further information, please visit: http://www.forestry.ubc.ca/students/g raduate/programs/master-of-international-forestry/or contact: Dr. Joleen Timko, Lecturer and Program Coordinator, Master of International Forestry, UBC Faculty of Forestry, Email: joleen.timko@ubc.ca.

\section{['O rure des ingénieurs forestiers du Q uébec (O IFQ)}

\section{D andonnée cycliste: une pre- 1 mière édition couronnée de succès}

Le vendredi 29 août dernier a eu lieu la première randonnée cycliste de l'Ordre des ingénieurs forestiers du Québec. Chaque année, l'Ordre organise un tournoi de golf qui permet d'amasser des fonds pour le financement d'activités pour les étudiants en foresterie de l'Université Laval. C'est dans le but de rallier un plus grand nombre de participants que l'Ordre a innové cette année en organisant cette première randonnée cycliste. La journée était idéale: le soleil était radieux, la température clémente et le vent était presque absent. Prêt d'une trentaine de cyclistes se sont réunis dans le stationnement du terrain de golf de Lotbinière vers $12 \mathrm{~h} 30$, enchantés par la température exceptionnelle et heureux de partager les kilomètres avec leurs confrères ingénieurs forestiers. Deux trajets étaient offerts, un trajet court de 60 kilomètres et un trajet long de 90 kilomètres, à travers les paysages agroforestiers de la région de Lotbinière. Une pause ravitaillement était offerte à mi-parcours aux participants et ceux-ci en ont profité pour se ressourcer, mais

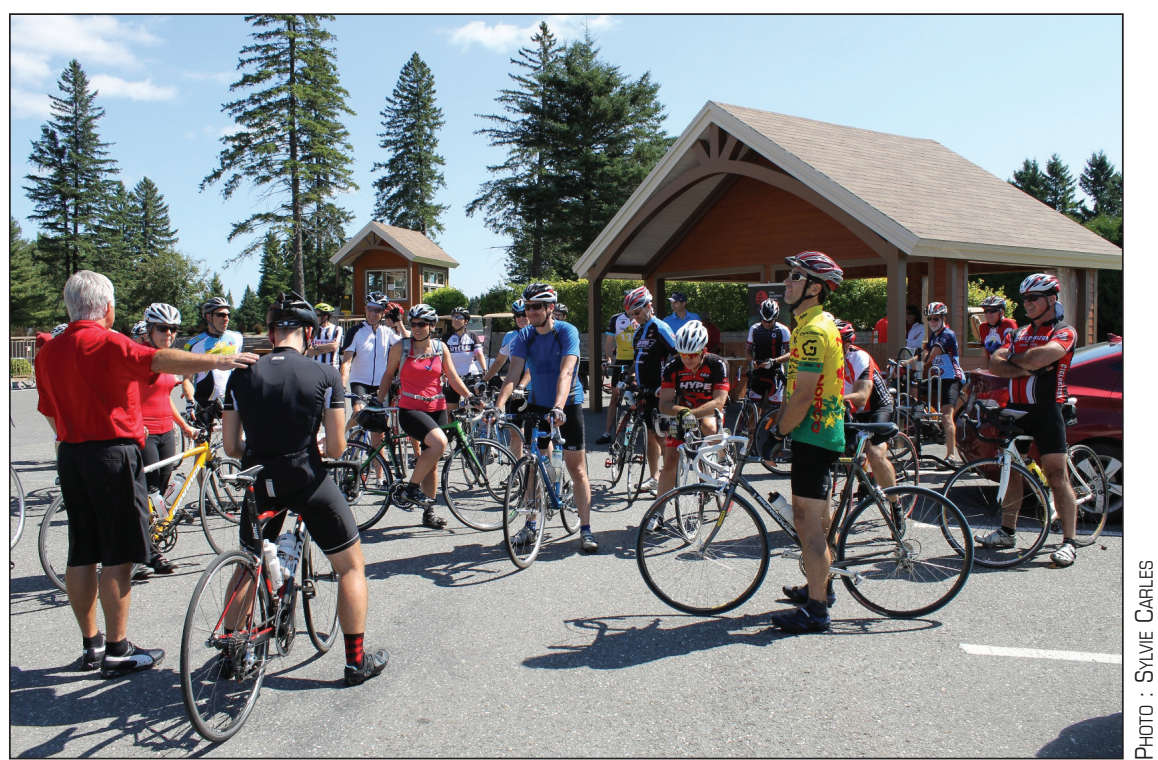

Une trentaine de participants se sont réunis pour le départ de la toute première randonnée cycliste de I'OIFQ.

surtout pour fraterniser davantage. Pour terminer la journée, les participants de la randonnée de vélo se sont joints aux golfeurs afin de partager le traditionnel banquet qui suit le tournoi de golf. L’ensemble des participants ont grandement apprécié cette nouvelle activité et nous sommes convaincus que ce sera le début d'une longue tradition.

L'Ordre aimerait remercier les bénévoles de la section Orléans de l'Institut forestier du Canada qui ont accepté d’organiser la pause ravitaillement. 